\title{
The On-line monitoring of the ALICE Muon Trigger at LHC
}

\section{Yongwook BAEK*}

Laboratoire de Physique Corpusculaire (LPC), Université Blaise Pascal, IN2P3/CNRS, Clermont-Ferrand, France

E-mail: yong.wook.baek@ecern.ch

\begin{abstract}
ALICE (A Large Ion Collider Experiment) [1] is the experiment dedicated to the study of ultrarelativistic heavy ion collisions at LHC. The ALICE Muon Spectrometer is mostly designed to detect muons from quarkonium and heavy flavour decays in a large range of forward pseudorapidity. Its performance should appropriate for an operation in the high multiplicity environment from central $\mathrm{Pb}-\mathrm{Pb}$ collisions as well as in the high rate of $\mathrm{pp}$ collisions, both requiring a fast and efficient trigger system. The Muon Trigger is based on four planes of Resistive Plate Chambers (RPCs) detectors each of them covering a total surface of about $36 \mathrm{~m}^{2}$. The RPCs are readout by means of 21,000 strips equipped with Front-End (FE) electronics. The trigger decisions are delivered by a fast electronics, organized in three levels: Local (receiving the signals from the FE), Regional and Global. The Global decision is sent to the ALICE Central Trigger Processor (CTP). To monitor detector performance and assure good data quality, an on-line monitoring tool, AMORE (Automatic MOnitoRing Environment), has been developed by ALICE. AMORE is interfaced to the DAQ software framework (DATE) and follows the publish-subscribe paradigm where a large number of batch processes execute detector specific analyses intro raw data samples and publish monitoring results on specialized servers. This poster aims at presenting the AMORE tool and the developments made for the Muon Trigger performance monitoring, including RPC detectors (strip scalers, strip patterns, strip multiplicities), algorithm decisions at all levels in the electronics, as well as overall trigger efficiency exploiting the redundancy of RPC planes.
\end{abstract}

XI Workshop on Resistive Plate Chambers and Related Detectors

5-10 February, 2012

Laboratori Nazionali di Frascati dell'INFN - Frascati (Rome) - Italy

${ }^{*}$ Speaker. 


\section{Introduction}

The ALICE experiment has been built to investigate nucleus-nucleus collisions at LHC energy. The aim of the muon spectrometer is to detect muons coming from the dimuonic decays of quarkonium states and semileptonic decays of heavy quarks, which are believed to be sensitive to the properties of the medium formed in heavy ion collisions.

The muon spectrometer [2] consists of five stations of tracking chambers to measure muon momentum by the help of the dipole magnet, two stations (MT1 and MT2) of trigger detectors to identify muons and absorbers to reduce the hadronic background. The muon spectrometer covers the pseudorapidity range $-4.0<\eta<-2.5$ with respect to the reference frame of ALICE.

\section{Muon Trigger System}

The Muon Trigger system selects muons from "signal" (unlike-sign muon pairs from decay of resonances, like-sign muon pairs for the background combinatorial studies and single muons from open heavy flavours). It is composed of two detector stations covering a large area (5.5 x 6.5 $\mathrm{m}^{2}$ ), of two planes each; each plane contains 18 single gap RPCs. The RPC detectors are made of two bakelite planes and can be operated either in streamer mode ( $\mathrm{HV} \sim 8 \mathrm{kV}$ with 50.5\% $\mathrm{Ar}+$ $41.3 \% \mathrm{C}_{2} \mathrm{H}_{2} \mathrm{~F}_{4}+7.2 \% \mathrm{iC}_{4} \mathrm{H}_{10}+1 \% \mathrm{SF}_{6}$ ) or avalanche mode ( $\mathrm{HV} \sim 10 \mathrm{kV}$ with $89.7 \% \mathrm{C}_{2} \mathrm{H}_{2} \mathrm{~F}_{4}+$ $10 \% \mathrm{C}_{4} \mathrm{H}_{10}+0.3 \% S F_{6}$ ). The Muon Trigger requires a time resolution better than $2 \mathrm{~ns}$, a spatial resolution $<1 \mathrm{~cm}$ and a rate capability $<100 \mathrm{~Hz} / \mathrm{cm}^{2}$.

Each RPC is readout by means of two perpendicular strip planes with strips of about 1, 2 and $4 \mathrm{~cm}$ width, projective with respect to the interaction point in each of the four planes. Each strip is equipped of Front-End electronics.

The trigger electronics has been designed to deliver, within $800 \mathrm{~ns}$, signlas to the CTP. The trigger decision procedure is organized in three levels: local, regional and global [4]. The DARC (Dimuon Arm Readout Controller) board is the interface with ALICE DAQ. The JTAG board is used to configure masks and LUT (Look-Up Table) [3] in the local trigger electronics. In addition, a pulse generator (FET for Front-End Test) is used to generate calibration events.

\section{Trigger Algorithm}

The signals from the FE electronics are sent to the 234 local trigger boards and the local trigger algorithm searches for single muon tracks coming from the interaction point, using the hit information of the 4 RPC detector planes. The local trigger decision requires a coincidence of hits from at least 3 out of 4 detector planes to define a track both in the bending plane (horizontal strips $\mathrm{X})$ and non-bending plane (vertical strips $\mathrm{Y}$ ).

The trigger algorithm running in local trigger boards allows to measure the track deviation related to the transverse momentum $p_{t}$, by means of the measurement of the track angle between the two stations, MT1 and MT2, with respect to the line pointing to the interaction center (Fig. 1). The muon trigger signals, above two $p_{t}$ cuts in parallel, are delivered to the ALICE CTP.

The maximum allowed deviation in strip units is of \pm 8 strips in bending plane corresponding $0.5 \mathrm{GeV} / \mathrm{c}$ and \pm 1 strip in non-bending plane, between MT1 and MT2. For the bending plane, the 


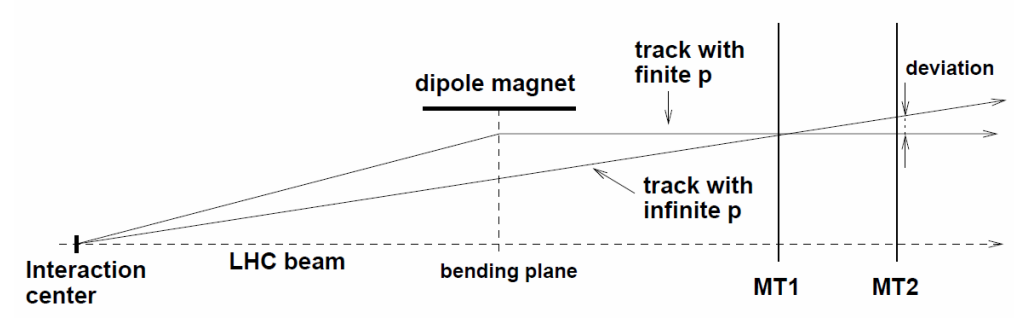

Figure 1: Principle of the muon trigger decision estimate (transverse momentum).

outputs of each local trigger boards are the track position (5 bits) in X on MT1 and the deviation (5 bits) between MT1 and MT2 including the sign of deviation. And the outputs for the non-bending plane are the trigger decision (1 bit) and the position (4 bits) in Y on MT1. The actual estimation of $p_{t}$ is performed through the LUT using each combination of outputs (X position, $\mathrm{X}$ deviation, $\mathrm{Y}$ trigger decision, $\mathrm{Y}$ position) and $2 p_{t}$ cuts (high $p_{t}$ and low $p_{t}$ ) are provided. The triggered track can be summarized with a word of 2 bits corresponding to the LUT response, and finally these 2 bits (for each $p_{t}$ cut) are sent to the regional trigger board. These 2 bits are:

- 00: No trigger

- 01: Trigger with up direction deviation

- 10: Trigger with down direction deviation

- 11: Trigger with no deviation

The 16 regional trigger boards make the regional trigger decisions for the single or di-muon (like-sign and unlike-sign) and are transfered to the global trigger board.

The global trigger board provides single and di-muon signals from the regional trigger information. The 6 trigger signals which are sent to CTP are as follows:

- SGL_LPT/SGL_HPT: Single muon Low $p_{t} /$ Single muon High $p_{t}$

- LS_LPT: at least 2 muons Low $p_{t}$ of like-sign

- LS_HPT: at least 2 muons High $p_{t}$ of like-sign

- US_LPT: at least 2 muons Low $p_{t}$ of unlike-sign

- US_HPT: at least 2 muons High $p_{t}$ of unlike-sign

\section{Monitoring Software Environment}

The aim of Monitoring software is to monitor data-taking status and detector status. This monitoring environment is based on Automatic MOnitoRing Environment (AMORE) which has been developed based upon the Data Acquisition (DAQ) [5] software framework, so-called the Data Acquisition and Test Environment (DATE).

The raw data structure of the Muon Trigger events is composed of DARC header (containing trigger occurrence, global trigger output and separator words), Regional header (DARC status word, regional word and inputs from the local boards) and Local board structure ( $\mathrm{X}$ and $\mathrm{Y}$ positions for 4 planes, local trigger decisions, separator words). 


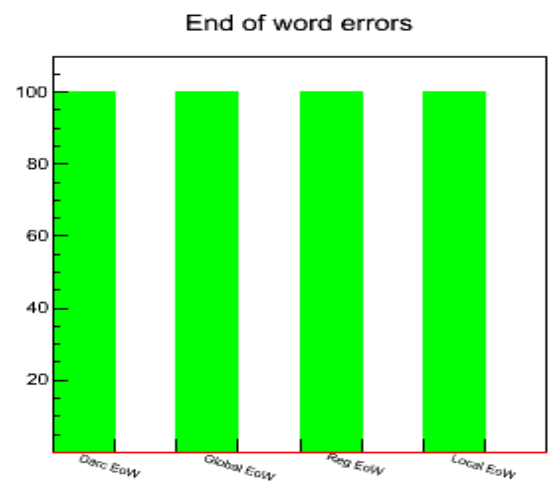

Figure 2: The raw data sturcture tests and its errors.

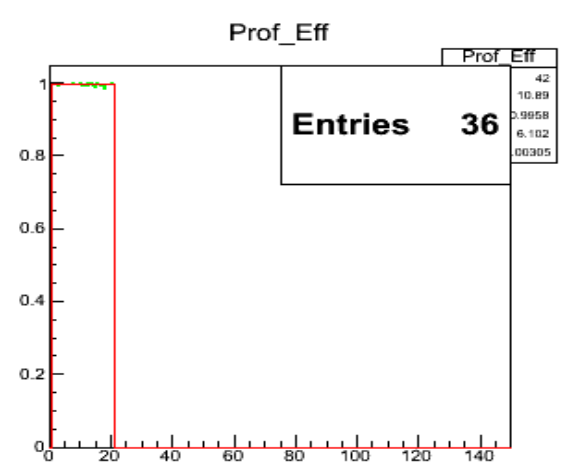

Figure 3: Time evolution of trigger efficiency ratio for the 44/34 coincidence of RPC planes.

The monitoring software for Muon Trigger system publishes many objects to be monitored in on-line. The objects reconstructed from the raw data are, in particular, the raw data structure and its size, the hit multiplicity, the detector efficiency, the 6 global trigger outputs and the scaler data.

Two different versions of the user interface for the monitoring software have been developed, shifter and expert version. The shifter version deals with the minimum possible number of objects that the shifter can easily check and recognize the detector status and its operation quality. On the other hand, the expert version treats many information in order to fully check the detector status.

\subsection{Shifter Version}

Two histograms are about the raw data structure and its errors (Fig. 2). The raw data size/event of Muon Trigger system is fixed to $6.6 \mathrm{kB}$. The multiplicity of fired electronics channels in case of calibration events offers the possibility to check the responses of the whole electronic chain from strips to global trigger board: expected multiplicities are $\sim 21 \mathrm{k}$ for strips, 234 trigger decisions for local, 16 for regional and 6 for global. Concerning the trigger efficiency, the time evolution of the ratio for the 44/34 (44 means 4 planes hit out of 4 possible, 34 means 3 planes hit out of 4 possible) coincidence of RPC planes has been implemented (Fig. 3). Normally, this ratio converges around some value and is sensitive to any change of detector operation condition. The global trigger outputs can be checked by comparing the actual decisions to the recalculated ones (from the $\mathrm{X}$ and Y hits in the 4 planes) at the monitoring Level. If any mismatch is found, the errors will show up on the plot of the 6 global trigger decisions. The global trigger outputs of physics events can also be checked in the same way as the calibration events.

\subsection{Expert Version}

The expert version is useful to have a closer look at the detector status and performance with more detailed information, especially, in case of trouble or any strange behaviour of detector. It contains more histograms than the shifter version.

The strip pattern display (Fig. 4) shows 234 histograms which correspond to all strips of the 234 local boards. With this display, we can see the distribution of hits on each plane and which local boards or strips do not respond. The total hit multiplicity per plane (for X and Y strips) 


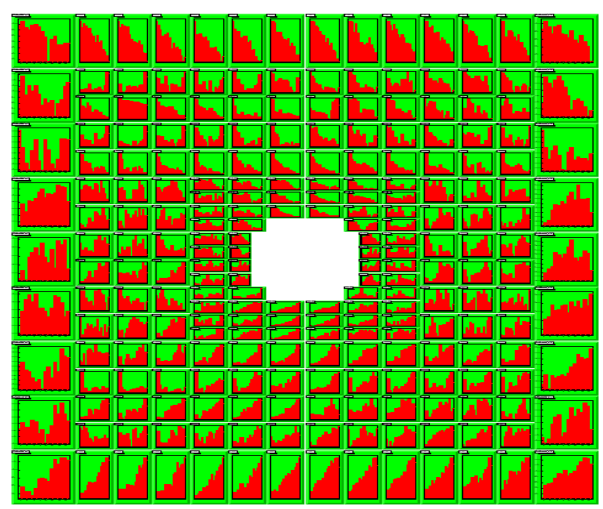

Figure 4: Strip pattern display showing 234 histograms of 234 local boards.

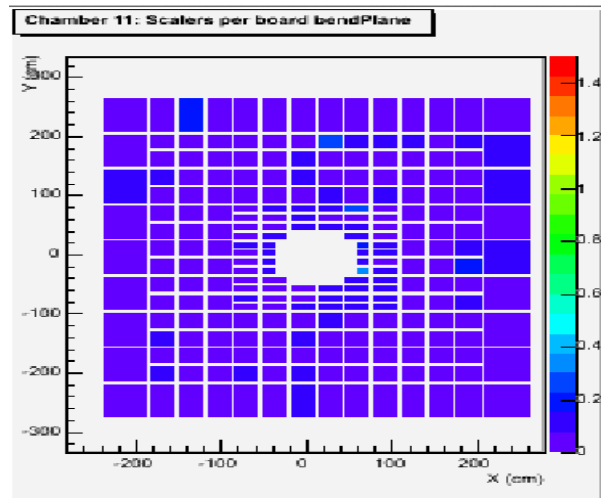

Figure 5: Strip scaler information to estimate the $\mathrm{RPC}$ single count rate in $\mathrm{Hz}$.

is also implemented. Concerning the local trigger algorithm test, not only LPT/HPT response per board and its errors, but also trigger multiplicity, triggered board, $\mathrm{X}$ position, $\mathrm{Y}$ position, deviations and errors can be checked. The algorithm test is very useful to check the malfunction of trigger electronics. The expert version allows to control the trigger efficiency stablility in terms of the ratio $44 / 34$ versus time and the ratio 44/34 versus local board, as well. During data taking, Muon Trigger system also performs measurements of RPC single count rate in $\mathrm{Hz}$ per strips, per local boards and RPCs (Fig. 5) using the strip scaler information.

\section{Conclusion}

Both on-line monitoring tools, i.e. the shifter and expert version, were developed particularly for the Muon Trigger system. They worked well in all data taking periods.

\section{References}

[1] ALICE Collaboration, JINST 3 (2008) S08002.

[2] ALICE Collaboration, Technical Design Report: Dimuon Forward Spectrometer, CERN/LHCC 99-22, CERN/LHCC 2000-046.

[3] G.A.Schuler and R.Vogt, Phys. Lett. B387 (1996) 181, [hep-ph/9606410].

[4] R. Arnaldi et al., IEEE Tran. Nucl. Sci. 51 (2004) 375.

[5] ALICE Collaboration : CERN-LHCC-2003-062. http://cdsweb. cern.ch/record/684651. 